\title{
Evidências da influência da vitamina $D$ com a fertilidade feminina: uma revisão sistemática
}

\author{
Evidence of the influence of vitamin D with female fertility: a systematic review \\ Evidencia de la influencia de la vitamina $D$ en la fertilidad femenina: una revisión \\ sistemática
}

Davi Candido Barbosa da Silva ${ }^{1 *}$, Jacqueline Carvalho dos Santos ${ }^{1}$, Nayane do Nascimento Costa1, Danielle Alice Vieira da Silva ${ }^{1}$, Ana Camila Lemos da Silva ${ }^{1}$, Jeane Belarmino da Silva', Jucileia Gomes Matias ${ }^{1}$, Priscila Monik Pereira de Lima', Alyne da Costa Araujo Ramalho', Priscilla Maria Bernardo da Silva'.

\section{RESUMO}

Objetivo: Constatar de que maneira a vitamina D pode influenciar na fertilidade feminina. Métodos: Para tanto, foi realizado uma revisão sistemática. As buscas foram feitas nas bases de dados da National Library of medicine (PubMed), Literatura Latino-Americana e do Caribe em ciências da saúde (LILACS) e Scientific Electronic Library (SCIELO) com as palavras chave Vitamin D AND Fertility. Resultados: Ao analisar os 11 artigos selecionados, constatou-se que a insuficiência e a deficiência de vitamina $D$ são condições frequentes na população feminina. Não foram encontradas de fato uma evidência direta de que o status de vitamina $D$ possa estar associada a fertilidade, mas foi visto que indiretamente os baixos níveis podem estar relacionados a várias patologias do sistema reprodutor feminino, como endometriose e baixa reserva ovariana, corroborando assim para uma possível infertilidade. Considerações Finais: Ressalta-se a importância de novas pesquisas para esclarecer as lacunas referente ao papel da vitamina $D$ e a fertilidade em mulheres.

Palavras-chave: Vitamina D, Fertilidade, Deficiência de vitamina D, Nutrientes.

\section{ABSTRACT}

Objective: Find out how vitamin D can influence female fertility. Methods: For this purpose, a systematic review was carried out. The searches were made in the databases of the National Library of medicine (PubMed), Latin American and Caribbean Literature in health sciences (LILACS) and Scientific Electronic Library (SCIELO) with the keywords Vitamin D AND Fertility. Results: When analyzing the 11 selected articles, it was found that vitamin D insufficiency and deficiency are frequent conditions in the female population. No direct evidence was found in fact that vitamin D status may be associated with fertility, but it was seen that indirectly low levels may be related to various pathologies of the female reproductive system, such as endometriosis and low ovarian reserve, thus corroborating for possible infertility. Final Considerations: The importance of new research is highlighted to clarify the gaps regarding the role of vitamin $\mathrm{D}$ and fertility in women.

Keywords: Vitamin D, Fertility, Vitamin D deficiency, Nutrients.

\section{RESUMEN}

Objetivo: Descubrir cómo la vitamina $D$ puede influir en la fertilidad femenina. Métodos: Para ello se realizó una revisión sistemática. Las búsquedas se realizaron en las bases de datos de la Biblioteca Nacional de Medicina (PubMed), Literatura Latino americana y del Caribe en Ciencias de la Salud (LILACS) y Biblioteca Electrónica Científica (SCIELO) con las palabras clave Vitamina D Y Fertilidad. Resultados: Al analizar los 11 artículos seleccionados, se encontró que la insuficiencia y la deficiencia de vitamina $D$ son afecciones frecuentes en la población femenina. De hecho, no se encontró evidencia directa de que el estado de la vitamina $\mathrm{D}$ pueda estar asociado con la fertilidad, pero se observó que los niveles indirectamente bajos pueden estar relacionados con diversas patologías del sistema reproductivo femenino, como la endometriosis y la baja reserva ovárica, corroborando así posible infertilidad. Consideraciones Finales: Se destaca la importancia de la nueva investigación para aclarar las brechas con respecto al papel de la vitamina D y la fertilidad en las mujeres.

Palabra clave: Vitamina D, Fertilidad, Deficiencia de vitamina D, Nutrientes.

${ }^{1}$ Centro Universitário Tiradentes (UNIT), Maceió - AL.

*E-mail: dcbs132@gmail.com 


\section{INTRODUÇÃO}

A vitamina $D$ é sintetizada fotoquimicamente na pele por meio de uma cascata de ativações que ocorrem no fígado e nos rins, e apenas uma pequena quantidade total do corpo é derivada de dieta e/ou suplementos. A literatura tem apontado que mais do que uma vitamina, essa molécula atua como um pseudo hormônio chave em vários processos metabólicos (DRESSLER N, et al., 2015; FUNG J, et al., 2017; LOPES VM et al., 2017).

Atualmente diversas funções da vitamina $D$ têm sido descritas, dentre as quais estão a homeostase do cálcio, fósforo e promoção a mineralização óssea, modulação da imunidade, síntese de interleucinas inflamatórias, controle da pressão arterial e, como participa da regulação dos processos de multiplicação e diferenciação celular, é atribuído também a ela papel anti oncogênico (CASTRO LCG, 2011; XU R, et al., 2020). Além dessas funções, no cenário atual, um conjunto de evidências observacionais e experimentais sugerem um papel potencialmente importante na função reprodutiva masculina, incluindo a qualidade do sêmen e o status androgênico. Isso é indicado por uma correlação positiva entre concentrações de vitamina $D$ e motilidade espermática e morfologia normal dos espermatozoides em homens inférteis.

O alcance dos órgãos-alvo da vitamina $D$ expandiu-se e o papel da vitamina $D$ na reprodução é destacado pela expressão do receptor da vitamina $D(V D R)$ e enzimas que metabolizam a vitamina $D$ no testículo e quando foi confirmada a presença de VDR nas células epiteliais glandulares do endométrio, células da granulosa, células epiteliais de Falópio e células do ovário (BOSDOU J, et al., 2019; NANDI A, et al., 2016).

Com relação a função reprodutiva feminina, tem-se que a deficiência de vitamina $D$ pode influenciar na fisiologia dos tecidos cervicais e uterinos, epitélios vaginais, células endometriais das trompas de falópio, ovários e glândulas pituitárias. Portanto, concentrações inadequadas de D-25OHD podem estar associadas a fatores de infertilidade, como anovulação crônica, endometriose e até câncer de mama (LOPES VM, et al., 2017). Os resultados de alguns estudos já sinalizam que níveis insuficientes de vitamina $D$ é comum entre as mulheres que apresentam infertilidade, variando de $21 \%$ a $31 \%$ em estudos realizados em países ocidentais e atingindo 75-99\% em estudos iranianos (VANNI V, et al., 2014; CHU J, et al., 2018).

Foi visto em modelos animais que a deficiência de vitamina $D$ está associada à função ovariana prejudicada. e diminuição da fertilidade. Entretanto, estudos em humanos limitaram-se a mulheres submetidas a tratamentos de fertilidade, como fertilização in vitro, e sugerem que as concentrações de vitamina $D$ séricas e no líquido folicular são preditivas do sucesso da fertilização in vitro por meio de efeitos na receptividade endometrial e na fertilização in vitro.

Devido ao seu papel como modulador imunológico, acredita-se que a vitamina $\mathrm{D}$ ajude a prevenir a perda da gravidez, facilitando a tolerância imunológica durante a gravidez. E por meio desses mecanismos, a vitamina D pode influenciar a fecundidade, o nascimento e a perda da gravidez (MUMFORD SL, et al., 2018). Diante do exposto objetivou-se constatar de que maneira o status de vitamina $D$ pode influenciar na fertilidade feminina.

\section{MÉTODOS}

Trata-se de um estudo exploratório descritivo, desenvolvido por meio de uma revisão sistemática que teve como foco investigativo realizar um levantamento bibliográfico que respondesse qual o papel da vitamina $D$ na fertilidade feminina in vivo.As buscas foram realizadas entre janeiro e fevereiro de 2020 e foram utilizados os bancos de dados eletrônicos das seguintes plataformas: National Library of medicine (PubMed), Literatura Latino-Americana e do Caribe em ciências da saúde (LILACS) e Scientific Electronic Library (SCIELO) de forma duplicada por acadêmicos de Nutrição. Para a realização das pesquisas nas bases de dados, foram estabelecidos descritores indexados na BVS utilizadas as seguintes combinações de descritores em inglês: Vitamin D AND Fertility.

O processo de seleção dos artigos seguiu uma adaptação do modelo proposto por Conforto EC, et al. (2011), que fez uso de filtros de seleção baseados nos critérios de inclusão do estudo, de forma que foram selecionados artigos originais, que relacionassem a vitamina $D$ e sua influência nos parâmetros de fertilidade feminina, publicados entre os anos de 2010 a 2020. Foram excluídos os artigos de revisão bibliográfica, os que não fizeram referência a vitamina $D$ e fertilidade, as dissertações e as teses, estudos in vitro e estudos feitos no público masculino. 
Total de artigos encontrados no PUBMED foram 134, LILACS 07 e SCIELO 03, totalizando 144 artigos. A seleção dos artigos foi realizada em três filtros: Primeiro filtro, exclusão após a leitura do título, foram 38 artigos de revisão e 52 artigos não associados aos descritores, restando 54 artigos. Em seguida, o segundo filtro de exclusão foi feito após a leitura do resumo, onde 23 artigos de revisão mostrado na metodologia e 02 artigos que envolviam animais, restando 29 artigos. O terceiro e último filtro foi feito após a leitura completa dos artigos, 18 não corresponderam ao objetivo final, portanto a revisão ficou contemplada com o total de 11 artigos.

\section{RESULTADOS}

Após a aplicação dos critérios, foram incluídos 11 artigos nesta revisão. Os estudos observados são ensaios clínicos que investigaram a associação da vitamina $D$ relacionando com a fertilidade. Observou-se que os ensaios clínicos foram feitos em diferentes países (Quadro 1). As populações estudadas eram homogêneas com mulheres com idades a partir de 18 anos. Todos os artigos selecionados estão indexados em revistas de qualis $\mathrm{A} 1-\mathrm{B} 2$.

Quadro 1 - Característica dos artigos selecionados - Relação entre a Vitamina D e Fertilidade. Estudo de revisão, 2020.

\begin{tabular}{|c|c|c|c|c|c|}
\hline Autores & Ano & Periódicos/Qualis & $\begin{array}{l}\text { Características } \\
\text { dos } \\
\text { Participantes }\end{array}$ & País & $\begin{array}{l}\text { Número de } \\
\text { referências }\end{array}$ \\
\hline $\begin{array}{l}\text { MOLLER UK, } \\
\text { et al. }\end{array}$ & (2012) & $\begin{array}{c}\text { European Journal of Clinical } \\
\text { Nutrition / A3 }\end{array}$ & 153 mulheres & Denmark & 46 \\
\hline $\begin{array}{l}\text { FABRIS AM, } \\
\quad \text { et al. }\end{array}$ & (2014) & $\begin{array}{c}\text { American Society for } \\
\text { Reproductive Medicine / A1 }\end{array}$ & 267 mulheres & $\begin{array}{l}\text { Madrid, Spain; e } \\
\text { United Arab } \\
\text { Emirates }\end{array}$ & 26 \\
\hline $\begin{array}{l}\text { DRESSLER N, } \\
\text { et al. }\end{array}$ & (2015) & $\begin{array}{l}\text { Gynecologic endocrinology and } \\
\text { reproductive medicine / A3 }\end{array}$ & 306 mulheres. & Germany & 40 \\
\hline $\begin{array}{l}\text { NEVILLE G, et } \\
\text { al. }\end{array}$ & $(2016)$ & $\begin{array}{l}\text { International Journal of } \\
\text { Gynecology and Obstetrics / A1 }\end{array}$ & $\begin{array}{l}75 \text { Homens e } \\
63 \text { Mulheres. }\end{array}$ & Dublin, Irlanda & 27 \\
\hline $\begin{array}{l}\text { LOPES VM, et } \\
\quad \text { al. }\end{array}$ & (2017) & $\begin{array}{l}\text { Arquivos de Endocrinologia e } \\
\text { Metabolismo / B2 }\end{array}$ & 369 Mulheres & Brasília, DF, Brasil & 35 \\
\hline $\begin{array}{l}\text { ABADIA L, et } \\
\text { al. }\end{array}$ & (2017) & $\begin{array}{l}\text { The American Journal of Clinical } \\
\text { Nutrition / A1 }\end{array}$ & 232 Mulheres & $\begin{array}{c}\text { Boston, } \\
\text { Massachusetts. }\end{array}$ & 35 \\
\hline FUNG J, et al. & (2017) & Fertility and Sterility / A1 & $\begin{array}{l}132 \text { Mulheres } \\
129 \text { Homens }\end{array}$ & $\begin{array}{l}\text { Boston, University } \\
\text { Park, e Líbano. }\end{array}$ & 47 \\
\hline $\begin{array}{l}\text { JUKIC AM, et } \\
\text { al. }\end{array}$ & (2018) & $\begin{array}{c}\text { The Journal of The North } \\
\text { American Menopause Society / } \\
\text { A2 }\end{array}$ & 561 Mulheres & $\begin{array}{l}\text { Chapel Hill, } \\
\text { Carolina do Norte, } \\
\text { Estados Unidos }\end{array}$ & 54 \\
\hline $\begin{array}{l}\text { MUMFORD } \\
\text { SL, et al. }\end{array}$ & (2018) & $\begin{array}{l}\text { Lancet -Diabetes Endocrinoly / } \\
\text { A1 }\end{array}$ & 1228 mulheres & Estados Unidos & 30 \\
\hline CHU J, et al. & (2019) & Reproductive Health / A2 & 500 Mulheres & Reino Unido & 34 \\
\hline $\begin{array}{l}\text { BEDNARSKA } \\
\text { e } \\
\text { CZERWIŃSKA } \\
\text { A, et al. }\end{array}$ & (2019) & Nutrients / A1 & 53 Mulheres & Basileia, Suíça. & 33 \\
\hline
\end{tabular}

Fonte: Silva DCB, et al., 2020.

Os dados apresentados no Quadro 2, integram ensaios clínicos onde mostram os resultados dos artigos revisados, inclusive autores, ano de publicação, desenho do estudo e principais desfechos. A dosagem da concentração sérica de D-25OHD considerada como adequada variou acordo com cada estudo. 


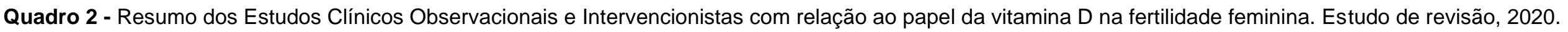

\begin{tabular}{|c|c|c|c|c|}
\hline Estudo & Objetivos & $\begin{array}{l}\text { Desenho de } \\
\text { Estudo }\end{array}$ & Avaliação & Resultados \\
\hline $\begin{array}{l}\text { MOLLER UK, } \\
\text { et al. (2012) }\end{array}$ & $\begin{array}{l}\text { Estudar os efeitos das } \\
\text { concentrações de D-25OHD } \\
\text { pré-concepção nas chances } \\
\text { de gravidez, bem como seus } \\
\text { efeitos durante a gravidez. }\end{array}$ & $\begin{array}{l}\text { Estudo de coorte } \\
\text { controlado de } \\
\text { base populacional } \\
\text { que ocorreu entre } \\
\text { outubro de } 2006 \text { a } \\
\text { janeiro de } 2008 \text {. }\end{array}$ & $\begin{array}{l}\text { As concentrações plasmáticas de D-25OHD foram } \\
\text { analisadas por cromatografia líquida de diluição de } \\
\quad \text { isótopos. } \\
\text { Não foi estabelecido pontos de corte para classificar os } \\
\text { níveis em adequados, insuficiente e deficiente, pois os } \\
\text { valores foram medidos por medianas. }\end{array}$ & $\begin{array}{l}\text { No início, a mediana de } \mathrm{D}-25 \mathrm{OHD} \text { foi de } 59 \mathrm{nmol} / \mathrm{L} \text {. } 31 \% \\
\text { das mulheres apresentavam } \mathrm{D}-25 \mathrm{OHD}<50 \mathrm{nmol} / \mathrm{L}, \\
\text { enquanto } 12 \% \text { apresentavam níveis acima de } 80 \mathrm{nmol} / \mathrm{L} \text {. } \\
\text { Os níveis de } \mathrm{D}-25 \mathrm{OHD} \text { não foram associados a chances } \\
\text { de concepção ou riscos de natimortalidade. }\end{array}$ \\
\hline $\begin{array}{l}\text { FABRIS AM, } \\
\text { et al. (2014) }\end{array}$ & $\begin{array}{l}\text { Investigar a correlação de D- } \\
25 O H D \text { sérica total } \\
\text { biodisponível e a taxa de } \\
\text { gravidez em receptores de } \\
\text { oócitos doados. }\end{array}$ & $\begin{array}{l}\text { Estudo } \\
\text { retrospectivo que } \\
\text { ocorreu entre } \\
\text { junho e dezembro } \\
\text { de } 2013 \text {. }\end{array}$ & $\begin{array}{l}\text { os níveis séricos de } \mathrm{D}-25 \mathrm{OHD} \text { foram determinados por } \\
\text { quimioluminescência. } \\
\text { Foram considerados como adequados os níveis de } \\
\text { vitamina } \mathrm{D}>30 \mathrm{ng} / \mathrm{mL} \text {, insuficiente de vitamina } D \text { entre } 20 \text { - } \\
\quad 30 \mathrm{ng} / \mathrm{mL} \text { e níveis de deficiência }<20 \mathrm{ng} / \mathrm{mL} \text {. } \\
\text { As pacientes foram divididas em } 3 \text { grupos: as que foram } \\
\text { submetidos a implantação de óvulos, as grávidas e as que } \\
\text { estavam se preparando para engravidar. }\end{array}$ & $\begin{array}{c}15,3 \% \text { da amostra estava com níveis adequados de } \\
\text { vitamina } D, 34,4 \% \text { tinham níveis insuficiente e } 50,2 \% \\
\text { tinham deficiência. } \\
\text { As taxas de implantação e gravidez não correlacionaram } \\
\text { com a vitamina } D \text { e não diferiram entre os três grupos } \\
\text { (70\%, } 69,9 \% \text { e } 73,9 \%) \text {. }\end{array}$ \\
\hline $\begin{array}{l}\text { DRESSLER } \\
\mathrm{N}, \text { et al. (2015) }\end{array}$ & $\begin{array}{l}\text { Investigar a prevalência de } \\
\text { deficiência de vitamina } D \text { em } \\
\text { mulheres inférteis e, identificar } \\
\text { os fatores de risco para } \\
\text { deficiência de vitamina } D \\
\text { além de especificar o impacto } \\
\text { das variações sazonais sobre } \\
\text { o status da vitamina D. }\end{array}$ & $\begin{array}{l}\text { Estudo de coorte } \\
\text { retrospectivo que } \\
\text { ocorreu entre } \\
\text { junho de } 2012 \text { e } \\
\text { dezembro de } \\
2013 .\end{array}$ & $\begin{array}{l}\text { Foram recrutadas mulheres inférteis de dois centros de } \\
\text { atendimentos. As medições de D-25OHD sérico foram } \\
\text { realizadas por meio de um ensaio total de vitamina } D \text {. } \\
\text { Foram mensurados peso e altura para posterior cálculo do } \\
\text { IMC. * }\end{array}$ & $\begin{array}{l}\text { 98,2\% dos pacientes no centro } 1 \text { e } 81,3 \% \text { das mulheres } \\
\text { com fertilidade comprometida no centro } 2 \text { apresentavam } \\
\text { níveis de vitamina D deficientes ou insuficientes. } \\
\text { Associou-se a deficiência de vitamina D os seguintes } \\
\text { fatores: IMC elevado (excesso de peso) e a exposição } \\
\text { limitada ao sol (inverno, primavera e outono) } \\
\text { Os níveis de vitamina D não variaram de acordo com a } \\
\text { idade ou com os distúrbios associados à infertilidade. }\end{array}$ \\
\hline $\begin{array}{l}\text { NEVILLE G, et } \\
\text { al. (2016) }\end{array}$ & $\begin{array}{l}\text { Avaliar o status da vitamina D } \\
\text { de homens e mulheres } \\
\text { submetidos à fertilização in } \\
\text { vitro e investigar associações } \\
\text { entre o status da vitamina D e } \\
\text { variáveis de fertilidade. }\end{array}$ & $\begin{array}{l}\text { Estudo } \\
\text { prospectivo } \\
\text { transversal que } \\
\text { ocorreu entre } \\
\text { janeiro e março } \\
\text { de } 2014\end{array}$ & $\begin{array}{c}\text { O soro D-25OHD foi medido por eletroquimioluminescência } \\
\text { a Pontos de corte: } \\
\text { <de } 30 \mathrm{nmol} / \mathrm{L} \text { indicado um risco especificado de } \\
\text { deficiência; } 30-50 \mathrm{nmol} / \mathrm{L} \text { foi o considerado como } \\
\text { insuficiente; > } 50 \mathrm{nmol} / \mathrm{L} \text { indicaram níveis suficientes de } \\
\text { vitamina } \mathrm{D} \text {. } \\
\text { A avaliação da fertilidade feminina foi feita através do } \\
\text { procedimento de coleta de óvulos e níveis hormonais anti- } \\
\text { müllerianos femininos. }\end{array}$ & $\begin{array}{c}\text { Entre as mulheres, não houve correlação significativa } \\
\text { entre } D-25 O H D \text { e hormônio anti-mülleriano }(p=0,63) \text { ou } \\
\text { número de óvulos colhidos }(p=0,198) \text { e ócitos } \\
\text { fertilizados ( } p=0,136) \text {. Não houve diferença estatística } \\
\text { nos níveis de } D-25 O H D \text { entre mulheres com e sem } \\
\text { gravidez em andamento }(p=0,22) .\end{array}$ \\
\hline $\begin{array}{l}\text { LOPES VM, et } \\
\text { al. } \\
(2017)\end{array}$ & $\begin{array}{l}\text { Determinar a prevalência de } \\
\text { hipovitaminose } D \text { em mulheres } \\
\text { brasileiras adultas e verificar } \\
\text { se as concentrações séricas } \\
\text { de vitamina } D \text { variavam de } \\
\text { acordo com o status de } \\
\text { fertilidade, idade ou causa da } \\
\text { infertilidade. }\end{array}$ & $\begin{array}{l}\text { Estudo de } \\
\text { delineamento } \\
\text { transversal } \\
\text { retrospectivo, que } \\
\text { ocorreu no ano de } \\
\quad 2012 \text {. }\end{array}$ & $\begin{array}{c}\text { A mensuração de D-25OHD foi realizada usando o método } \\
\text { de quimioluminescência. } \\
\text { Para avaliar a fertilidade adotou-se a investigação de: } \\
\text { Baixa reserva ovariana - contagem folicular antral; foi } \\
\text { investigado a presença de síndrome dos ovários } \\
\text { policísticos - com base nos critérios de consenso de } \\
\text { Roterdâ; }\end{array}$ & $\begin{array}{c}\text { Entre as } 369 \text { mulheres avaliadas, } 81,1 \% \text { apresentaram } \\
\text { insuficiência e } 32,0 \% \text { apresentaram deficiência de } \\
\text { vitamina } D \text {. Os pacientes inférteis e os grupo de controle } \\
\text { não apresentaram diferenças significativas na } \\
\text { prevalência de deficiência de vitamina } D(30,2 \% \text { vs. } 35 \% \text {, } \\
\text { respectivamente; } p=0,33) \text { ou nas concentrações médias } \\
\text { de } D-25 O H D(24,3 \pm 7,9 \mathrm{ng} / \mathrm{mL} \text { vs. } 23,8 \pm 8,7 \mathrm{ng} / \mathrm{mL} \text {, } \\
\text { respectivamente; } p=0,51) .\end{array}$ \\
\hline
\end{tabular}

REAS / EJCH | Vol.12(12) | e4878 | DOI: https://doi.org/10.25248/reas.e4878.2020 Página 4 de 10 


\begin{tabular}{|c|c|c|c|c|}
\hline Estudo & Objetivos & $\begin{array}{l}\text { Desenho de } \\
\text { Estudo }\end{array}$ & Avaliação & Resultados \\
\hline $\begin{array}{l}\text { ABADIA L, et } \\
\text { al. } \\
(2017)\end{array}$ & $\begin{array}{l}\text { Examinar a associação entre } \\
\text { concentrações de } \mathrm{D}-25 \mathrm{OHD} \text { e } \\
\text { o resultado em mulheres } \\
\text { submetidas a tecnologias de } \\
\text { reprodução assistida. }\end{array}$ & $\begin{array}{l}\text { Estudo de coorte } \\
\text { prospectivo que } \\
\text { ocorreu entre } \\
2006 \text { e } 2014 \text {. }\end{array}$ & $\begin{array}{l}\text { As concentrações séricas de D-25OHD foram medidas por } \\
\text { um ensaio imunoenzimático. } \\
\text { Não foram estabelecidos pontos de corte para classificar } \\
\text { os níveis em adequados, insuficientes ou suficientes. A } \\
\text { análise foi feita por mediana. As mulheres foram pré- } \\
\text { tratadas com contraceptivos orais; }\end{array}$ & $\begin{array}{c}\text { As concentrações séricas medianas de } \mathrm{D}-25 \mathrm{OHD} \text { foram } \\
\text { de } 86,5 \mathrm{nmol} / \mathrm{L} \text {. } 91 \% \text { dos participantes consumiram } \\
\text { polivitamínicos. As concentrações séricas de } \mathrm{D}-25 \mathrm{OHD} \\
\text { estavam positivamente relacionadas à taxa de } \\
\text { fertilização, mas não relacionadas à probabilidade de } \\
\text { gravidez ou pós gestação. A taxa de fertilização ajustada } \\
\text { para mulheres nos quartis crescentes de soro } \mathrm{D}-25 \mathrm{OHD} \\
\text { foram de } 0,62 \text { (IC } 95 \%: 0,51,0,72), 0,53 \text { (IC } 95 \%: 0,43 \text {, } \\
0,63 \text { ), } 0,67 \text { (IC } 95 \%: 0,56,0,76 \text { ) e } 0,73 \text { (IC } 95 \%: 0,63 \text {, } \\
0,80 \text { ), respectivamente (tendência com } p=0,03 \text { ), } \\
\text { indicando que quanto maior os valores de vitamina } \mathrm{D} \text {, } \\
\text { maior chances de fertilização. }\end{array}$ \\
\hline $\begin{array}{l}\text { FUNG J, et al. } \\
\quad(2017)\end{array}$ & $\begin{array}{l}\text { Avaliar o papel da ingestão de } \\
\text { vitamina D e dos níveis } \\
\text { séricos na concepção de } \\
\text { gravidez e pós gestação. }\end{array}$ & $\begin{array}{l}\text { Estudo de coorte } \\
\text { prospectivo que } \\
\text { ocorreu entre } \\
\text { maio de } 2006 \text { e } \\
\text { junho de } 2012 \text {. }\end{array}$ & $\begin{array}{c}\text { A D-25OHD foi dosada pelo método de espectrometria. } \\
\text { O status da vitamina } D \text { foi determinado como suficiente ou } \\
\text { em risco de inadequação ou deficiência quando os níveis } \\
\text { séricos de } D-25 O H D \text { foram } \geq 50 \text { nmol/L ou< } 50 \text { nmol/L, } \\
\text { respectivamente. } \\
\text { A ingestão de vitamina } D \text { foi avaliada usando a avaliação } \\
\text { de três recordatórios alimentares. } \\
\text { Os dados de ingestão alimentar foram calculados com o } \\
\text { apoio do software NDSR } \\
\text { A ingestão de vitamina } D \text { foi comparada com a EAR }{ }^{\star} \text {. }\end{array}$ & 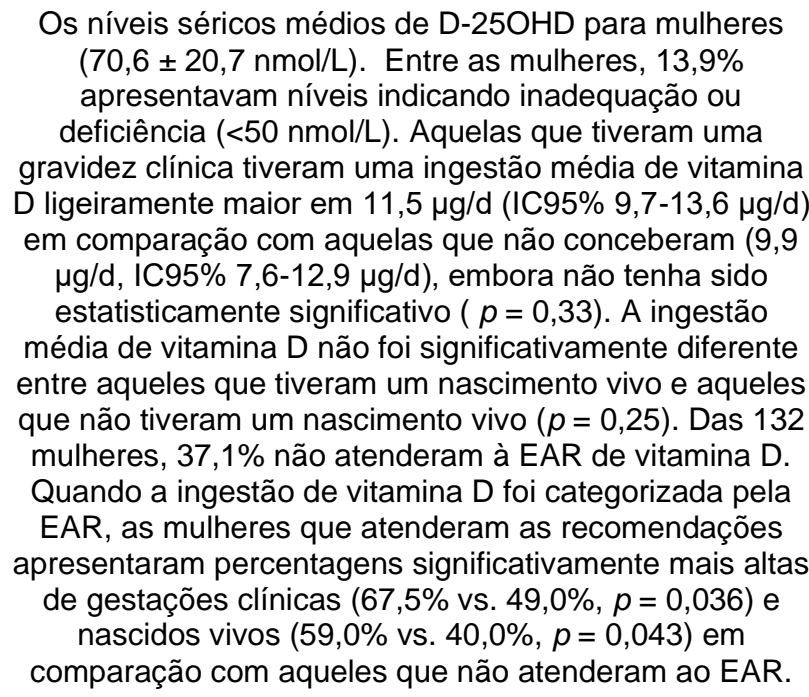 \\
\hline $\begin{array}{l}\text { JUKIC AM, et } \\
\text { al. (2018) }\end{array}$ & $\begin{array}{l}\text { Examinar as associações } \\
\text { entre D-25OHD e } \\
\text { biomarcadores da reserva } \\
\text { ovariana em uma grande } \\
\text { amostra comunitária de } \\
\text { mulheres. }\end{array}$ & $\begin{array}{l}\text { Estudo de coorte } \\
\text { prospectivo que } \\
\text { ocorreu entre } \\
2008 \text { e } 2016 .\end{array}$ & $\begin{array}{l}25(\mathrm{OH}) \mathrm{D} 3 \text { e } 25(\mathrm{OH}) \mathrm{D} 2 \text { foram quantificados por } \\
\text { cromatografia líquida - espectrometria de massa em } \\
\text { tandem. Não foram estabelecidos pontos de corte para } \\
\text { classificar os níveis em adequados, insuficientes ou } \\
\text { suficientes. A análise foi feita por média e mediana. }\end{array}$ & $\begin{array}{l}\text { A média de D-25OHD foi de } 36 \mathrm{ng} / \mathrm{mL} \text {. Os níveis de } \mathrm{D} \text { - } \\
25 \mathrm{OHD} \text { não se correlacionaram com hormônio anti } \\
\text { mulleriano, hormônio folículo estimulante ou inibina-B. } \\
\text { Para desfechos dicotômicos, houve uma tendência à } \\
\text { insuficiência de } \mathrm{D}-25 \mathrm{OHD}(<30 \mathrm{ng} / \mathrm{mL}) \text { em associação } \\
\text { com baixo AMH }(<0,7 \mathrm{ng} / \mathrm{mL})(\text { odds ratio }[\mathrm{IC} 95 \% \text { : } 1,8 \\
[0,9-4]) .\end{array}$ \\
\hline
\end{tabular}

REAS / EJCH | Vol.12(12) | e4878 | DOI: https://doi.org/10.25248/reas.e4878.2020 


\begin{tabular}{|c|c|c|c|c|}
\hline Estudo & Objetivos & $\begin{array}{l}\text { Desenho de } \\
\text { Estudo }\end{array}$ & Avaliação & Resultados \\
\hline $\begin{array}{l}\text { MUMFORD } \\
\text { SL, et al. } \\
(2018)\end{array}$ & $\begin{array}{l}\text { Avaliar as associações entre } \\
\text { concentrações séricas de pré- } \\
\text { concepção D-25OHD e } \\
\text { fecundabilidade, perda na } \\
\text { gravidez e parto em mulheres } \\
\text { com fecundidade comprovada, } \\
\text { bem como o efeito da D- } \\
25 O H D \text { sérica medida no } \\
\text { início da gestação e ao } \\
\text { decorrer da gestação. }\end{array}$ & $\begin{array}{c}\text { Estudo de coorte } \\
\text { prospectivo que } \\
\text { ocorreu entre } 15 \\
\text { de junho de } 2007 \\
\text { a } 15 \text { de julho de } \\
2011 .\end{array}$ & $\begin{array}{l}\text { Análise secundária de um coorte prospectivo do estudo } \\
\text { Effects of Aspirin in Gestation and Reproduction, um } \\
\text { estudo clínico multicêntrico, randomizado em bloco, duplo- } \\
\text { cego e controlado por placebo. } \\
\text { O status de vitamina } D \text { nas mulheres foram classificados } \\
\text { como vitamina } D \text { insuficiente }(<75 \mathrm{nmol} / \mathrm{L}) \text { ou suficiente } \\
(\geq 75 \mathrm{nmol} / \mathrm{L}) \text {. }\end{array}$ & $\begin{array}{l}1191 \text { mulheres tinham dados disponíveis sobre as } \\
\text { concentrações precoces de D-25OHD. } 555(47 \%) \\
\text { mulheres foram classificadas como tendo concentrações } \\
\text { suficientes }(\geq 75 \text { nmol / L) e } 636(53 \%) \text { como tendo } \\
\text { concentrações insuficientes }(<75 \mathrm{nmol} / \mathrm{L}) \text {. } \\
\text { Mulheres com níveis suficiente D-25OHD tiveram maior } \\
\text { probabilidade de engravidar do que as mulheres com } \\
\text { concentrações insuficientes, sendo a diferença } \\
\text { estatisticamente comprovada }(p<0,05)\end{array}$ \\
\hline $\begin{array}{l}\text { CHU J, et al. } \\
(2019)\end{array}$ & $\begin{array}{l}\text { Investigar a associação entre } \\
\text { vitamina e } \\
\text { D e resultados do tratamento } \\
\text { reprodutivo em mulheres } \\
\text { submetidos a tratamentos } \\
\text { reprodutivos assistidos }\end{array}$ & $\begin{array}{l}\text { Estudo de coorte } \\
\text { prospectivo que } \\
\text { ocorreu entre } \\
\text { setembro de } 2013 \\
\text { a setembro } 2015 \text {; }\end{array}$ & $\begin{array}{l}\text { O D-25OHD foi dosada por um método de extração } \\
\text { líquido-líquido usando alta espectrometria de massa por } \\
\text { cromatografia líquida sensível; } \\
\text { Adotou-se os seguintes pontos de corte: deficiente em } \\
\text { vitamina } D(<\text { menor que } 50 \text { nmol/L), insuficiente em } \\
\text { vitamina } D(50-75 \mathrm{nmol} / \mathrm{L}) \text { e suficientes em vitamina } D \\
\text { (>maior que } 75 \mathrm{nmol} / \mathrm{L}) \text {. As participantes mulheres foram } \\
\text { submetidos à estimulação ovariana com hormônio } \\
\text { estimulador folicular da hipófise recombinante. }\end{array}$ & $\begin{array}{c}\text { A deficiência de vitamina } D \text { foi encontrada em } 53,2 \% \text { das } \\
\text { participantes e a insuficiência de foi encontrada em } \\
30,8 \% \text { e } 16,0 \% \text { estavam suficientes de vitamina } D \text {. } \\
\text { As taxas de nascimentos vivos para mulheres } \\
\text { deficientes, insuficientes e suficientes foram } 23,2 \% \text {, } \\
27,0 \% \text { e } 37,7 \% \text {, respectivamente }(p=0,04) \text {. } \\
\text { Após o ajuste para os principais fatores prognósticos as } \\
\text { taxas de nascimentos vivos para mulheres deficientes } \\
\text { em vitamina } D \text {, insuficientes e suficientes foram } 24,3 \text {, } \\
27,1,34,4 \% \text { respectivamente, sem diferença estatística } \\
\text { entre os grupos }(p=0,25) \text {. }\end{array}$ \\
\hline $\begin{array}{l}\text { BEDNARSKA } \\
\text { e } \\
\text { CZERWIŃSK } \\
\text { A A, et al. } \\
\text { (2019) }\end{array}$ & $\begin{array}{l}\text { Identificar se alterações nas } \\
\text { concentrações de vitamina D } \\
\text { influenciam no status do } \\
\text { hormônio Anti-Mulleriano. }\end{array}$ & $\begin{array}{l}\text { Estudo de coorte } \\
\text { prospectivo que } \\
\text { ocorreu no ano de } \\
2017 .\end{array}$ & $\begin{array}{l}\text { Amostras de sangue e testes hormonais. } \\
\text { As medições laboratoriais foram realizadas com um } \\
\text { imunoanalisador eletroquimioluminescente. } \\
\text { Os níveis de hormônio Anti-Mulleriano e vitamina D no } \\
\text { Fluido Folicular foram medidos de acordo com os limites } \\
\text { de quantificação do ensaio em } 3,0 \text { e } 0,1 \mathrm{ng} / \mathrm{ml} \text {. }\end{array}$ & $\begin{array}{c}\text { Houve relação da vitamina } D \text { com a fertilidade, } \\
\text { determinado uma diferença estatisticamente significante } \\
(p<0,05) \text { nos níveis totais de vitamina } D \text { e hormônio anti- } \\
\text { Mulleriano no soro entre os semestres, mostrando que } \\
\text { quanto maior a deficiência de vitamina } D \text { maior as } \\
\text { manifestações de infertilidade. }\end{array}$ \\
\hline
\end{tabular}

Legenda: *D-25OHD: 25-hidroxivitamina D; IMC: Índice de Massa Corporal; NDSR: Nutrition Data System for Research; EAR: Necessidade Média Estimada; IC95\%: Intervalo de Confiança; AMH: Hormônio anti-Mulleriano.

Fonte: Silva DCB, et al., 2020.

REAS / EJCH | Vol.12(12) | e4878 | DOI: https://doi.org/10.25248/reas.e4878.2020 Página 6 de 10 


\section{DISCUSSÃO}

\section{Deficiência de vitamina $D$ no público feminino em idade fértil}

A deficiência de vitamina $D$ já é considerada como uma manifestação endêmica em diversas regiões do mundo e atinge com maior expressividade os grupos considerados de risco: idosos, negros, lactentes. A deficiência de vitamina $D$ é um problema de saúde global e está associada a inflamação, função autonômica, distúrbios autoimunes, dor crônica, câncer e distúrbios vasculares e neurológicos, além de seus papéis esqueléticos (KAMBOJ P, et al., 2018).

Mais de dois bilhões de pessoas sofrem de deficiência de vitamina $D$, sendo que a deficiência de vitamina $\mathrm{D}$ também aumenta os riscos de complicações maternas e fetais. Nos últimos anos o estudo do status dessa vitamina em mulheres com idade fértil têm ganhado destaque, uma vez que parece haver uma relação intrínseca entre os níveis séricos e o processo de fertilidade (CHU J, et al., 2019).

Deficiência de vitamina $D$ é definida como concentrações de $25(\mathrm{OH}) \mathrm{D}<20 \mathrm{ng} / \mathrm{mL}$ ( $50 \mathrm{nmol} / \mathrm{L}$ ), enquanto insuficiência de vitamina D como concentrações de $25(\mathrm{OH}) \mathrm{D} 20-30 \mathrm{ng} / \mathrm{mL}(50-75 \mathrm{nmol} / \mathrm{L})$. A prevalência de deficiência de vitamina $D$ varia de 8 a $90 \%$ na Europa (atingindo> 50\% nas populações da Europa Ocidental) e de 14 a $89 \%$ na América do Norte. Várias organizações recomendaram diferentes níveis de ingestão de vitamina $D$ para suas respectivas populações-alvo. Ingestão recomendada de vitamina $D$ geralmente varia entre 5 e $20 \mu \mathrm{g} /$ dia, dependendo da idade e do estado fisiológico (BOSDOU J, et al., 2019).

Nesta revisão constatou-se altas prevalências de deficiência entre as mulheres avaliadas, sendo o maior percentual $53 \%$ encontrado no estudo de Chu J, et al. (2019), e o menor 32\% na pesquisa de Lopes VM, et al. (2017). Coincidentemente o estudo que mostrou o menor percentual foi realizado no Brasil, país esse que tem maior incidência de raios solares durante o ano em comparação ao realizado no Reino Unido. Apesar de acometer um número elevado de mulheres em idade fértil, um outro estudo enfatiza a escassez de pesquisas publicadas descrevendo a importância da vitamina $D$ no público feminino para que se estime uma prevalência global (BURKE NL, et al., 2019).

Os dados encontrados identificam a vitamina $\mathrm{D}$ como um ponto - chave no sucesso reprodutivo, sugerindo que os níveis de D-25OHD, quando insuficientes ou deficientes, podem estar associados com várias causas de infertilidade. Ultimamente tem sido, feitos numerosos estudos que investigaram o efeito potencial do status materno da vitamina $D$ nas complicações da gravidez, como pré-eclâmpsia e parto prematuro, além de resultados prematuros e do nascimento, incluindo baixo peso ao nascer e nanismo no primeiro ano de gravidez (MUMFORD SL, et al., 2018).

\section{Fatores associados à deficiência de Vitamina D}

Os resultados de vários modelos de regressão usados por Dressler N, et al. (2015), sustentam a hipótese de que o excesso de peso e o menor tempo de exposição solar se associam ao risco aumentado de deficiência da vitamina $D$.

A radiação ultravioleta solar (UV) -B (UVB; comprimentos de onda de 290 a $315 \mathrm{~nm}$ ) estimula a síntese de vitamina D3 a partir do 7-desidrocolesterol na epiderme da pele, fomentando com isso a síntese de mais de 90\% da vitamina endógena. Sendo assim, radiação inadequada ou absorção cutânea insuficiente de UVB solar são algumas das principais causas de depleção dessa vitamina (BARREA L, et al., 2017). Segundo Nouri S, et al. (2017), isso explica o fato de maiores prevalências de insuficiência no status de vitamina $D$ serem encontradas em países onde o inverno é mais longo e indivíduos que se expõe com pouca frequência ao sol.

Há também outros mecanismos possíveis que podem explicar a deficiência deste pseudo hormônio na vigência do excesso de peso, como a menor ingestão por indivíduos obesos, menor exposição da pele à luz solar, devido à menor atividade ao ar livre quando comparado a indivíduos saudáveis, diminuição da absorção intestinal devido a procedimentos bariátricos e/ou disbiose intestinal e prejuízos nas etapas de hidroxilação devido a alterações em sinalizadores (VIMALESWARAN N, et al., 2013).

É importante salientar que apesar do consumo alimentar não ser o fornecedor principal de vitamina $D$ endógena, sua oferta não deve ser negligenciada, como indicado no estudo de Fung $\mathrm{J}$ et al. (2017), onde maiores adequações frente a EAR garantiram melhores desfechos. 
As principais fontes de vitamina $D$ são exposição a luz solar, peixe oleoso, produtos dietéticos fortificados e suplementos nutricionais. Suplementos de vitamina D estão disponíveis como prescrições e como preparações de balcão. Como prescrições, estão disponíveis cápsulas de vitamina $\mathrm{D}_{2}$ (ergocalciferol) que contêm 50.000 Ul de vitamina $D_{2}$ por cápsula. O outro produto de prescrição é uma forma líquida, que contém $8000 \mathrm{Ul} / \mathrm{ml}$ de vitamina $\mathrm{D}_{2}$.

Preparações multivitamínicas estão disponíveis sem receita e contêm $400 \mathrm{UI}$ de vitamina $\mathrm{D}_{2}$ ou $\mathrm{D}_{3}$. A vitamina $\mathrm{D}_{3}$ em produtos que contêm $400 \mathrm{UI}, 800 \mathrm{UI}, 1000 \mathrm{UI}$ e 2000 UI também está disponível sem receita. Os alimentos com maior teor de vitamina D são óleo de peixe (400-1000 UI / colher de óleo), salmão capturado (600-1000 Ul / $100 \mathrm{~g}$ ), enguia (1200 Ul / $100 \mathrm{~g}$ ), arenque em óleo (400-800 Ul / $100 \mathrm{~g}$ ), sardinha (300 UI / $100 \mathrm{~g}$ ), salmão em lata (300-600 UI / $100 \mathrm{~g}$ ), arenque em lata (250 UI / $100 \mathrm{~g}$ ), atum em lata (230 UI / $100 \mathrm{~g}$ ), cogumelos shiitake (100 UI / $100 \mathrm{~g}$ ), gema de ovo (20-50 Ul / 1 gema de ovo), leite de vaca (0,4-1,2 $\mathrm{UI} / 100 \mathrm{~mL}$ ), leite materno (1,5-8 UI / $100 \mathrm{~mL}$ ) e queijo (7-28 UI / $100 \mathrm{~g})$ (DOVNIK, A; MUJEZINOVIĆ, F. 2018).

\section{Vitamina $D$ e aspectos da fertilidade feminina: Endometriose}

$A$ vitamina $D$ possui efeitos biológicos que vão muito além do sistema músculo esquelético, já sendo evidenciado sua importância no manejo de agravos crônicos não transmissíveis e de forma mais recente na saúde feminina, sobretudo ao que se refere a fertilidade (MARINO R e MISRA M, 2019).

Lopes VM, et al. (2017), reiteram que a vitamina D desempenha um papel importante em vários processos metabólicos e pode influenciar a fisiologia reprodutiva das mulheres. Os autores reforçam ainda que concentrações séricas inadequadas da mesma podem estar associadas a fatores já validados na literatura como de risco para infertilidade, como anovulação crônica e endometriose.

A endometriose é crescimento de glândulas endometriais e estroma fora da cavidade uterina, um distúrbio inflamatório benigno comum que afeta 5 a $10 \%$ das mulheres em idade reprodutiva, as principais características clínicas, incluindo dor pélvica, dismenorreia, dispareunia e infertilidade. A patogênese ainda não está bem estabelecida, mas parece que uma resposta imune e inflamatória alterada permite a sobrevivência de implantes endometriais (VOULGARIS N, et al., 2017). Além disso foi visto em um estudo de revisão que a vitamina $D$ pode ser relacionada à patogênese da endometriose devido aos seus efeitos imunomoduladores e anti-inflamatórios (SAYEGH L, et al., 2014).

Os nutrientes e vitaminas essenciais diários podem modular os processos imunológicos e inflamatórios que são alterados em mulheres com endometriose. Por exemplo, além de seus efeitos bem conhecidos na homeostase do fosfato de cálcio, a D-25OHD tem um forte potencial de modulação imune e efeitos na proliferação e diferenciação celular, tanto nos tipos normais quanto nos malignos. Tanto a endometriose quanto a deficiência de vitamina $D$ têm sido associadas a doenças autoimunes, como doença de Crohn e artrite reumatóide (AKYOL A, et al., 2016).

De forma sistematizada, pode-se dizer que a associação entre vitamina $D$ e endometriose é baseada nos seguintes achados; o endométrio humano expressa Receptor de Vitamina D (RVD) e 1-alfahidroxilase(enzima), podendo ser um possível local de síntese extra renal e ação da vitamina D (CERMISONI GC, et al., 2018). E seus efeitos imunomoduladores; macrófagos, células dendríticas e linfócitos expressam o RVD, enquanto sua forma ativa 1,25 (OH) D, atua através do RVD que induz a destruição de agentes microbianos e inibe a apresentação de antígenos e a maturação das células dendríticas (LI N, et al., 2017).

Estudos mostraram dados que ligam a vitamina $\mathrm{D}$ e a endometriose emergem principalmente de estudos observacionais e mostram resultados conflitantes. Vários estudos não notaram nenhuma diferença entre os níveis séricos de vitamina $D$ em pacientes com endometriose e indivíduos saudáveis. No entanto, a expressão de VDR e 1a-hidroxilase foi maior no endométrio e ovários de mulheres com endometriose em comparação com indivíduos saudáveis, embora essa diferença tenha sido estatisticamente significante apenas para a 1ahidroxilase, implicando uma maior produção local do metabólito ativo calcitriol e / ou um aumento da ação da vitamina D.

Por outro lado, dados publicados recentemente mostram uma relação inversa entre o nível de vitamina $D$ e a endometriose, pois mulheres com maior nível de D-25OHD tiveram um risco $24 \%$ menor de desenvolver endometriose do que mulheres com níveis mais baixos (HOLLY RH, et al., 2013; VOULGARIS N, et al., 2017). 


\section{Vitamina $D$ e aspectos da fertilidade feminina: Biomarcadores de reserva ovariana}

A reserva ovariana é um parâmetro da função ovariana, representando a quantidade e a qualidade dos folículos ovarianos. Pode ser usado para prever o risco de infertilidade feminina, o sucesso do tratamento da infertilidade, a duração da vida reprodutiva e a idade da menopausa. Embora diminua gradualmente com o aumento da idade, há uma alta variabilidade interindividual na reserva ovariana entre mulheres de idade semelhante, e a taxa de declínio na reserva ovariana varia amplamente entre mulheres em idade reprodutiva.

Quando o número de folículos ovarianos é reduzido a um limiar crítico, ocorre a menopausa. Além das influências genéticas e da idade na reserva ovariana, acredita-se que fatores ambientais modifiquem a taxa de declínio na reserva ovariana. O impacto da nutrição nos processos que induzem a atrofia folicular, incluindo estresse oxidativo e inflamação, apoia a possibilidade de que fatores nutricionais possam influenciar a reserva ovariana e a idade da menopausa (MOSLEHI N, et al., 2017).

No artigo publicado por Jukic AM, et al. (2018), constatou-se que uma reserva ovariana reduzida (baixa quantidade de oócitos) está associada a uma resposta fraca à estimulação ovariana entre as mulheres que procuram tratamento de fertilidade. A reserva ovariana reduzida com a menopausa precoce subsequente não apenas limita o tempo de vida reprodutiva da mulher, mas também tem sido associada ao aumento do risco de osteoporose, doença cardiovascular e mortalidade por todas as causas.

Os ovários iniciantes resultam em declínio tanto na quantidade, quanto na qualidade dos folículos e oócitos ovarianos. O ovário envelhecido produz pequenas quantidades de hormônios granulares, como Hormônio Anti-mulleriano (HAM), 10 e inibição B11, o que leva a um aumento do Hormônio Folículo-estimulante (FSH) da fase folicular precoce (GASPARIN AA, et al., 2015).

Um dos biomarcadores mais importantes produzidos pelas células da granulosa, que desempenha um papel importante na foliculogênese, é o hormônio anti-Mülleriano (AMH). O AMH é um membro da superfamília de fator de crescimento transformador beta, também conhecida como substância inibidora de Müller (MIS). Está envolvido na regressão dos ductos Müllerianos em embriões do sexo masculino em mamíferos e aves.

Em mamíferos do sexo feminino, sua produção começa somente após a 36a semana de gestação e é secretada exclusivamente por células granulosas de folículos ovarianos independentes das gonadotrofinas. Sua secreção começa quando os folículos são recrutados do pool primordial para se tornarem folículos primários, atingem um pico no estágio pré-antral / antral pequeno e diminuem quando atingem o tamanho final e o estado de diferenciação disponíveis para seleção pelo hormônio folículo-estimulante da hipófise (FSH) (MORIDI I, et al., 2020).

Uma revisão conduzida na Polônia levantou a hipótese de que a vitamina $D$ tem um efeito direto na produção de HAM e, portanto, pacientes com maiores concentrações dela são capazes de manter sua reserva ovariana por mais tempo (GRZECHOCINSKA B, et al., 2013). Dennis N, et al. (2012) e Fabris AM, et al. (2017), confirmam que a vitamina $D$ pode ser um regulador positivo da produção de HAM ou relatam que seus níveis não estão relacionados à reserva de óvulos ou à resposta ovariana após estimulação da mesma, ressaltando seu papel no processo de fecundação.

Complementando as informações citadas, um estudo de revisão examinou o papel deste nutriente na fisiologia ovariana e suas implicações para a reprodução e concluiu que sua deficiência acarreta condições adversas à fertilidade feminina, pois o HAM inibe a transição folicular. Esse efeito repressivo é mediado por receptores de HAM tipo II altamente específicos (AMHR2). Assim, um maior nível de HAM é responsável por suprimir a maturação folicular, neste caso o papel da vitamina D é inibir a AMHR2, facilitando assim a maturação do folículo (MERHI Z, et al., 2014).

\section{CONSIDERAÇÕES FINAIS}

A insuficiência e a deficiência de vitamina $D$ são condições frequentes na população feminina. Não foi encontrada de fato uma evidência direta de que seu status possa estar associada a fertilidade, mas foi visto que indiretamente os baixos níveis desse pseudo hormônio pode estar relacionados a várias patologias do sistema reprodutor feminino, corroborando assim para uma possível infertilidade. Sendo assim, novas pesquisas devem ser estimuladas para esclarecer as lacunas referente ao papel desse nutriente na fertilidade. 


\section{REFERÊNCIAS}

1. ABADIA L, et al. Serum 25-hydroxyvitamin $D$ concentrations and treatment outcomes of women undergoing assisted reproduction. The American journal of clinical nutrition, 2016; 104:729-35.

2. AKYOL A, et al. Efficacies of vitamin $D$ and omega-3 polyunsaturated fatty acids on experimental endometriosis. Taiwanese journal of obstetrics \& gynecology, 2016; 55:835-839.

3. BARREA L, et al. Low serum vitamin D-status, air pollution and obesity: A dangerous liaison. Reviews in endocrine \& metabolic disorders, 2017; 18:207-214.

4. BEDNARSKA-CZERWINSKA A, et al. Vitamin D and Anti-Müllerian Hormone Levels in Infertility Treatment: The ChangePoint Problem. Nutrients, 2019; 11:1053.

5. BOSDOU J et al. Vitamin D and Obesity: Two Interacting Players in the Field of Infertility. Nutrients, 2019; 11:1455.

6. BURKE NL, et al. Determinants of vitamin $\mathrm{D}$ status among Black and White low-income pregnant and non-pregnant reproductive-aged women from Southeast Louisiana. BMC Pregnancy Childbirth, 2019; 19:111.

7. CASTRO LCG. O sistema endocrinológico vitamina D. Arq Bras Endocrinologia Metabologia, São Paulo, 2011; 55(8): 566575.

8. CERMISONI GC, et al. Vitamin D and Endometrium: A Systematic Review of a Neglected Area of Research. International journal of molecular sciences, 2018; 19:2320.

9. $\mathrm{CHU} \mathrm{J} \mathrm{et} \mathrm{al.} \mathrm{Vitamin} \mathrm{D} \mathrm{and} \mathrm{assisted} \mathrm{reproductive} \mathrm{treatment} \mathrm{outcome:} \mathrm{a} \mathrm{systematic} \mathrm{review} \mathrm{and} \mathrm{meta-analysis.} \mathrm{Human}$ reproduction, 2018; 33:65-80.

10. CHU J, et al. Vitamin D and assisted reproductive treatment outcome: a prospective cohort study. Reproductive health, 2019; 16:106.

11. CONFORTO EC, et al. (2011). Roteiro para revisão bibliográfica sistemática: aplicação no desenvolvimento de produtos e gerenciamento de projetos. In Anais Congresso Brasileiro de Gestão de Desenvolvimento de Produtos, Porto Alegre, 2011; 8.

12. DENNIS NA, et al. The level of serum anti-Müllerian hormone correlates with vitamin $D$ status in men and women but not in boys. The Journal of Clinical Endocrinology \& Metabolism, 2012; 97:2450-2455.

13. DOVNIK A, MUJEZINOVIĆ F. The Association of Vitamin D Levels with Common Pregnancy Complications. Nutrients, 2018; 10(7): 867.

14. DRESSLER N, et al. BMI and season are associated with vitamin D deficiency in women with impaired fertility: a two-centre analysis. Archives of gynecology and obstetrics, 2016; 293:907-914.

15. FABRIS AM, et al. Impact of circulating levels of total and bioavailable serum vitamin $D$ on pregnancy rate in egg donation recipients. Fertility and sterility, 2014; 102:1608-1612.

16. FABRIS AM, et al. Impact of vitamin $D$ levels on ovarian reserve and ovarian response to ovarian stimulation in oocyte donors. Reproductive biomedicine online, 2017; 35:139-144.

17. FUNG J, et al. Association of vitamin D intake and serum levels with fertility: results from the Lifestyle and Fertility Study. Fertility and sterility, 2017; 108:302-311.

18. GASPARIN AA, et al. Hormônio anti-Mülleriano como preditor de reserva ovariana em pacientes lúpicas: uma revisão. Rev. Bras. Reumatol, São Paulo, 2015; 55:363-367.

19. GRZECHOCINSKA B, et al. The role of vitamin D in impaired fertility treatment. Neuroendocrinol Lett, 2013; 34:756-762.

20. HOLLY RH, et al. Missmer, Dairy-Food, Calcium, Magnesium, and Vitamin D Intake and Endometriosis: A Prospective Cohort Study, American Journal of Epidemiology, 2013; 177:420-430.

21. IRANI $M, M E R H I Z$. Role of vitamin $D$ in ovarian physiology and its implication in reproduction: a systematic review. Fertility and sterility, 2014; 102(2): 460-468.

22. JUKIC AM, et al. 25-Hydroxyvitamin D (25(OH)D) and biomarkers of ovarian reserve. Menopause,2018; 25:811-816.

23. KAMBOJ $P$, et al. Prevalence of hypovitaminosis $D$ in India \& way forward. The Indian journal of medical research, 2018; 148:548-556.

24. LI N, et al. Women with recurrent spontaneous abortion have decreased $25(\mathrm{OH})$ vitamin $\mathrm{D}$ and VDR at the fetal-maternal interface. Brazilian journal of medical and biological research, 2017; 50:6527.

25. LOPES VM et al. Highly prevalence of vitamin D deficiency among Brazilian women of reproductive age. Arch. São Paulo. Endocrinol. Metab, 2017;61:21-27.

26. MARINO R, MISRA M. Extra-Skeletal Effects of Vitamin D. Nutrients, 2019; 11(7): 1460.

27. MERHI Z, et al. Vitamin $D$ alters genes involved in follicular development and steroidogenesis in human cumulus granulosa cells. The Journal of Clinical Endocrinology \& Metabolism, 2014; 99:1137-1145.

28. MOLLER UK, et al. Effects of $25 \mathrm{OHD}$ concentrations on chances of pregnancy and pregnancy outcomes: a cohort study in healthy Danish women. European journal of clinical nutrition, 2012; 66: 862-868.

29. MOSLEHI N, et al. Current Evidence on Associations of Nutritional Factors with Ovarian Reserve and Timing of Menopause: A Systematic Review. Advances in nutrition, 2017; 8:597-612.

30. MORIDI I, et al. The Association between Vitamin D and Anti-Müllerian Hormone: A Systematic Review and MetaAnalysis. Nutrients, 2020; 12:1567.

31. MUMFORD SL, et al. Association of preconception serum 25-hydroxyvitamin D concentrations with livebirth and pregnancy loss: a prospective cohort study. The lancet. Diabetes \& endocrinology, 2018;

32. NANDI A, et al. Is there a role for vitamin D in human reproduction?. Hormone molecular biology and clinical investigation, 2016; 25:15-28.

33. NEVILLE G, et al. Vitamin D status and fertility outcomes during winter among couples undergoing in vitro fertilization/intracytoplasmic sperm injection. International journal of gynaecology and obstetrics: the official organ of the International Federation of Gynaecology and Obstetrics, 2016; 135:172-176.

34. NOURI S, et al. Seasonal variations of vitamin $D$ and its relation to lipid profile in Iranian children and adults. Journal of health, population, and nutrition, 2017; 36: 1-7.

35. SAYEGH L, et al. Vitamin D in endometriosis: a causative or confounding factor?. Metabolism: clinical and experimental, 2014; 63:32-41.

36. VANNI V, et al. Vitamin D and assisted reproduction technologies: current concepts. Reproductive biology and endocrinology: RB\&E, 2014; $12: 47$.

37. VIMALESWARAN K, et al. Causal relationship between obesity and vitamin D status: bi-directional Mendelian randomization analysis of multiple cohorts. PLoS Med, 2013; 10: 100-383.

38. VOULGARIS N, et al. Vitamin D and aspects of female fertility. Hormones, 2017; 16:5-21.

39. XU R, et al. Association of vitamin D status with coronary artery disease in postmenopausal women. Medicine, 2020; 99:1944. 